\title{
A Study on Protocol for Efficient Multimedia Data Transmission Scheme in Wireless Satellite Network
}

\author{
Sung-Gyu Kim, Jinho Lee and Byungjoo Park* \\ Department of Multimedia Engineering, Hannam University \\ 133 Ojeong-dong, Daeduk-gu, Daejeon, Korea \\ sgkim@hnu.kr,jin@iitels.com,bjpark@hnu.kr* \\ *Correspondent Author: Byungjoo Park* (bjpark@hnu.kr)
}

\begin{abstract}
Recently, satellite networks that provide network services have received much attention. With satellite networks, it is possible to communicate at higher speeds with more distant areas, and to efficiently manage the employment amount of packets. As a result, many service providers are interested in services over satellite networks. However, satellite networks have many problems such as high transmission time and high packet loss rate. In addition, High Return Trip Time (RTT) also occurs. These characteristics make it difficult to provide high-quality services through satellite networks. Therefore, in this paper, a method to provide high - quality service to users by transmitting data more efficiently through satellite network is proposed.
\end{abstract}

Keywords: Transmission Control Protocol, Performance Enhancing Proxies, satellite link

\section{Introduction}

In the past, wired networks are accounted for the largest percentage of the Internet market. However, the proportion of wireless networks is widely increasing due to the development and diffusion of technologies such as smart phones, notebooks, and tablet PCs. Recently, satellite networks have attracted much attention. Satellite networks can provide high-speed communication between more distant areas and facilitate high-capacity packet management. However, satellite networks are characterized by longer transmission times and high packet loss rates.

Most of the data that are currently transmitted through the network is managed using the Transmission Control Protocol (TCP). However, TCP was developed based on the low bandwidth of the early days of the Internet. Network technology has eventually evolved, and most of the network services that are currently offered are high-quality contents that require higher bandwidth. TCP is also based on wired networks and is not suitable for wireless network environments. When using the TCP in a satellite network, there are many limitations due to problems such as higher link error, transmission delay, and bandwidth asymmetry. In other words, there are many difficulties in providing a high-quality service in the satellite network with the currently used TCP protocol. To overcome this, we use Performance Enhancing Proxies (PEP) in the satellite network. However, since the PEP manages the satellite network with only one RTT value, it is very difficult to find the complications since the whole RTT is too long. In this paper, a protocol that can provide high quality service to the users by solving the problems that can occur in the PEP of the satellite network is proposed.

Received (July 3, 2017), Review Result (August 30, 2017), Accepted (September 9, 2017) 


\section{Related Studies}

\subsection{TCP}

Figure 1 shows the packet transmission method of TCP and User Datagram Protocol (UDP). TCP uses an acknowledgment (ACK) message to ensure that packets are sent from the source to the destination. The ACK message is a message that the destination responds to the sender that it has received the packet. In this way, if a packet is lost, reliable transmission of the packet is possible by sending the packet again at the source. UDP, on the other hand, does not divide data into packets at the source and reassemble at the destination. The sender transmits the data regardless of whether the packet was properly received at the destination. This has the advantage of being simple to implement and fast. However, UDP can cause a best-effort error. In addition, there is a problem that an application needs to process if a packet retransmission or a problem of a reverse order of a packet arises. For this reason, most networks currently use TCP.

TCP uses four congestion control algorithms, Congestion Avoidance, Fast Retransmission, Slow Start, and Fast Recovery, to control the size of the Congestion Window for ACK response. In case of using TCP using the congestion control algorithm in a satellite network, high transmission delay, transmission error, and bandwidth asymmetry occur and performance is degraded.

- Satellite networks have a higher transmission error rate than other networks. Path attenuation due to relatively long transmission distance, and rainfall attenuation due to the weather condition. Accordingly, even if an excellent error correction algorithm is used, a high transmission error rate occurs.

- In the TCP congestion control process, the high transmission delay of the satellite link hinders the increase of the data rate.

- Asymmetry of the satellite network causes congestion of ACK.
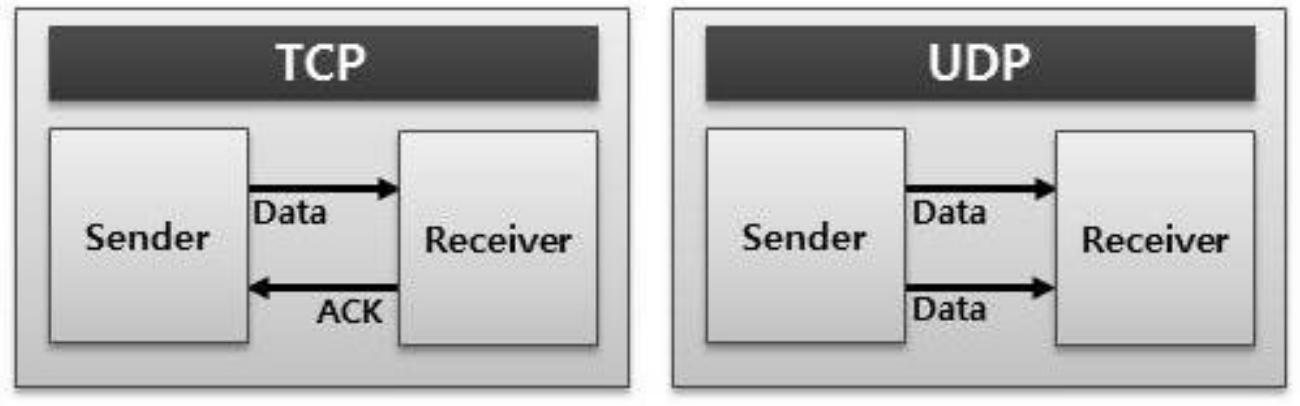

Figure 1. Packet Transmission Method of TCP and UDP

\subsection{PEP}

TCP is currently used as a transport layer protocol for applications used in the majority of Internet and intranet environments. However, control over TCP occurs when protocol performance of a specific environment and other upper layers is limited by link characteristics. The quality of service provided through the network depends on how efficiently the network is managed. That is, the satisfaction of the user is determined according to how efficiently the network is managed by using the protocol.

PEP has been developed to overcome the problems caused by the nature of satellite networks. That is, the PEP operating at the transport layer is defined in RFC 3135 to manage the satellite network environment more efficiently. TCP is a protocol optimized for wired network environment. In other words, TCP enables efficient communication in 
a wired network with a low packet loss rate. However, performance degrades in wireless networks where high packet loss rates occur due to noise and interference. Satellite networks may also suffer from performance degradation when using conventional TCP due to the long transmission delay time, high packet loss rate, and large bandwidth delay product. Several other methods can be used by the PEP to improve network application performance.

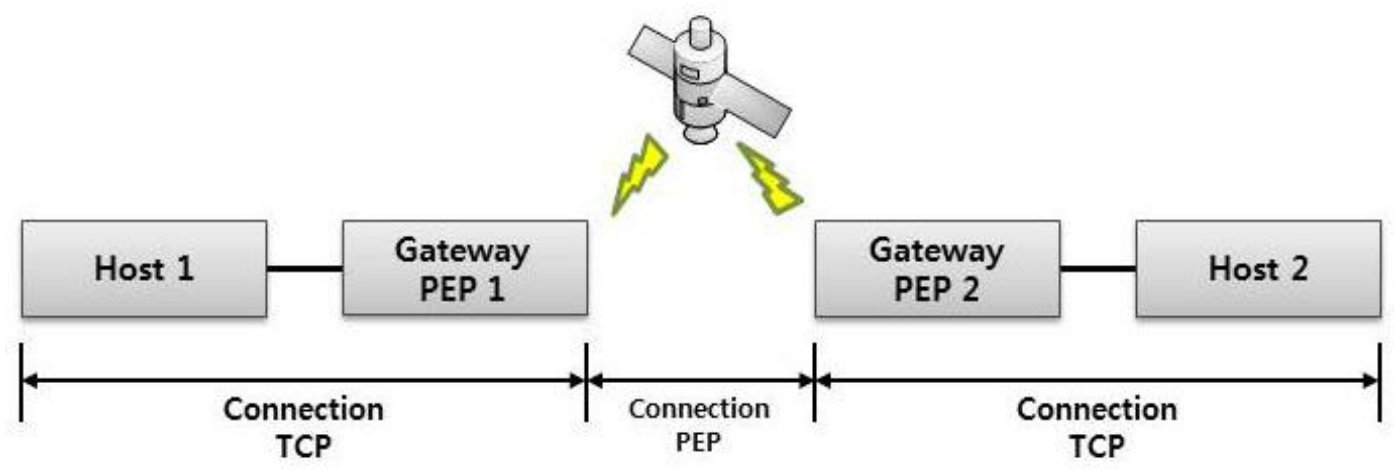

Figure 2. Basic PEP Protocol Components

Common PEP mechanisms include local TCP authentication and retransmission techniques as well as traffic compression. Distributed PEPs will also use various forms of tunneling for per-traffic traffic and can be designed to maintain a persistent TCP connection even during power outages between subnets to improve the robustness of the detected client application. Additional mechanisms provide priority based on multiplexing of protocols and special-purpose protocols. PEP has been proposed to overcome the above-mentioned problems and enable more efficient communication in satellite networks. Figure 2 shows the basic structure of the PEP.

\subsection{PEP operation procedure}

Figure 3 shows the operation procedure of the PEP. PEP manages the satellite network environment based on end-to-end communication. The data of the server is transmitted to the satellite transmission center through the Internet by dividing the address and various information of the sender and the receiver in the TCP / IP layer into packet units. This packet is sent to the PEP terminal by bundling the packets divided through the TCP / IP layer. In the PEP, a series of processes for authentication and billing, data compression, and security are sent to the Enhanced TCP layer, which is divided into packets according to the satellite network. After that, the transceiver that transmits the packet through the satellite network and receives the packet is divided into the structure suitable for the wired network environment through the reverse order of the previous step. This packet is sent to the user over the Internet. The PEP manages the network through the satellite network with one RTT value. 


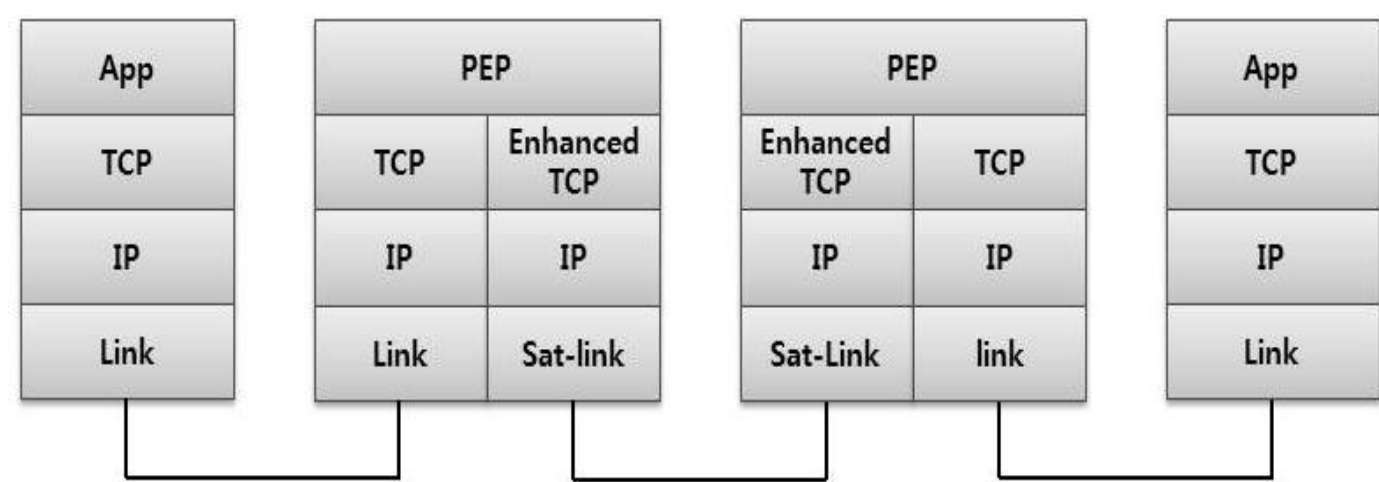

Figure 3. PEP Operation Procedure

Therefore, even if a loss occurs in a satellite network environment, only one RTT needs to be managed, so the performance decline rate is smaller than that of PEPsal. However, since the PEP manages all the sections as one RTT, it is hard to find the point where the failure occurs and the whole RTT becomes very long.

\section{Perspicacious PEP (P-PEP)}

\subsection{P-PEP}

Using P-PEP proposed in this paper, more efficient data transmission is possible in satellite network. The proposed scheme modified satellite and existing PEP.

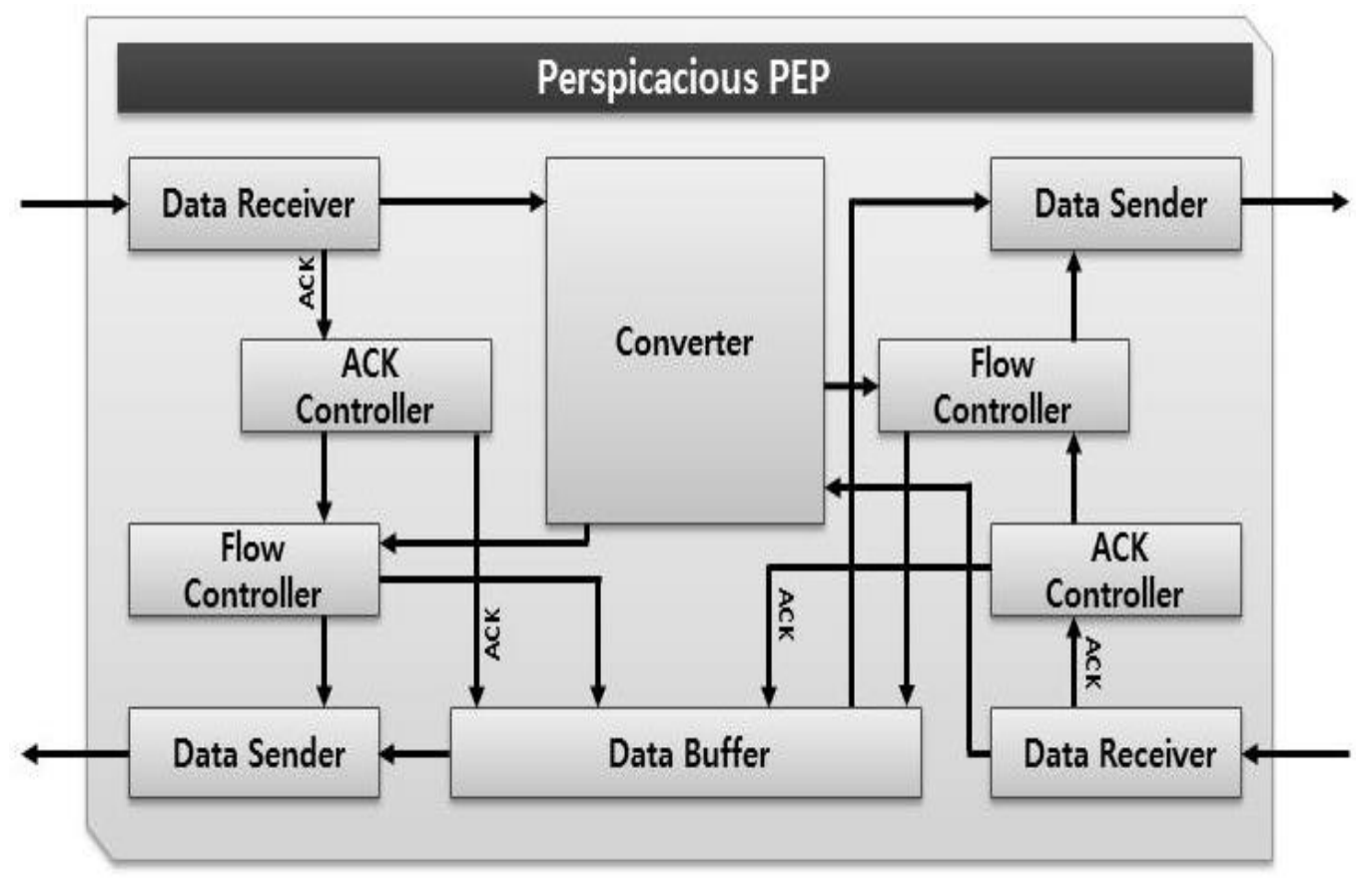

Figure 4. P-PEP Architecture 


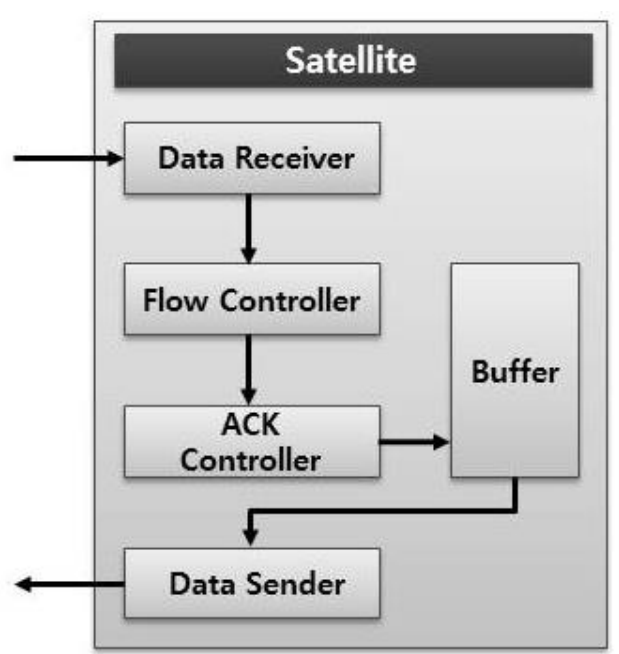

Figure 5. Satellite Architecture

P-PEP consists of Data Receiver, Data Sender, ACK Controller, Flow Controller, Data Buffer, Converter. Figure 4 shows the architecture of P-PEP proposed in this paper. The Data Receiver divides the transmitted data into packets and ACKs. Data Sender is responsible for transmitting data. The ACK Controller manages the ACK message for each data. The flow controller controls the data transmission. Also, the arrival time of the ACK message is estimated through the RTT value. If the ACK message is not received after the estimated time, the packet is retransmitted. The Data Buffer is used to temporarily store packets that have been received from the source and have not yet received an ACK message. Converters convert TCP protocol type packets into Enhanced TCP protocol type.

The satellite consists of Data Receiver, Data Sender, Flow Controller, ACK Controller and Buffer. Each configuration plays the same role as in the P-PEP described above. Figure 5 shows the architecture of the satellite network.

\subsection{P-PEP Operation Procedure}

Figure 6 shows the basic structure of P-PEP proposed in this paper.

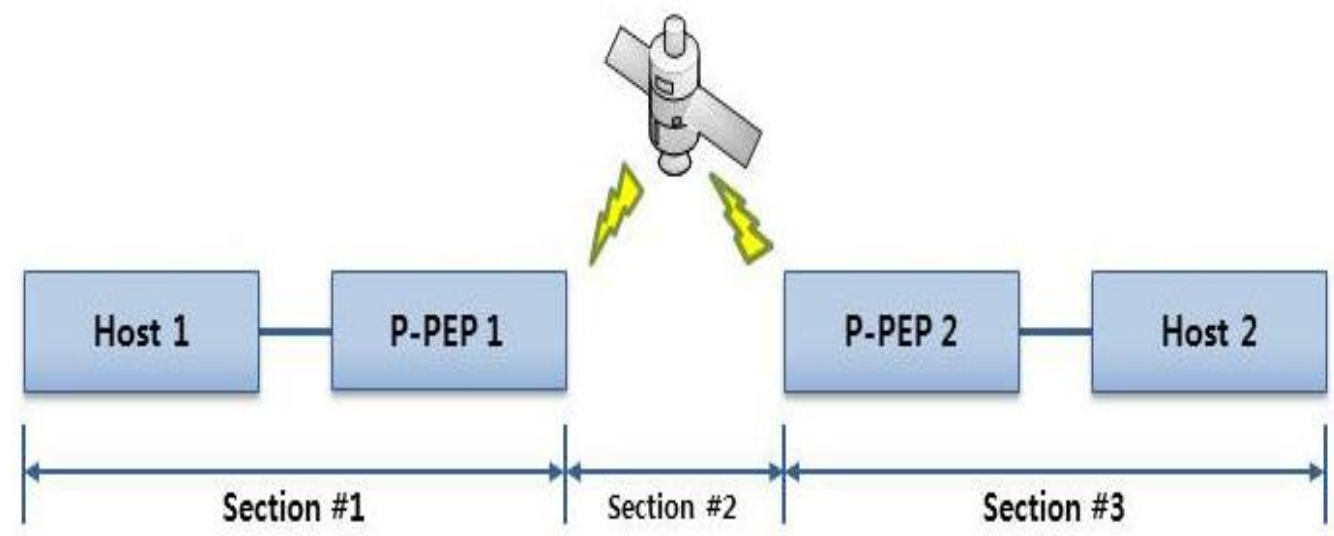

Figure 6. Basic P-PEP Protocol Components 


\section{- $\quad$ Section \#1}

Transmission Host Host 1 transmits data to P-PEP 1 through TCP congestion control algorithm. The P-PEP 1 receives the packet and sends it to Converter, converts it into Enhanced TCP structure, generates ACK message from ACK Controller, and sends it to Host 1 . The flow controller measures the ACK message time for the corresponding packet through the RTT value and temporarily stores the packet in the data buffer. The Data Sender then sends the packet to the satellite. After transmitting data to the satellite, the P-PEP must receive an ACK message to the satellite. Flow Controller measures RTT value and measures ACK arrival time for each packet. If the ACK message is normally received, the packet stored in the flow controller and data buffer is deleted. This can prevent over-flow problems. If the ACK message is not received even after the measured time has elapsed, the Flow Controller determines that the connection has failed and retransmits the packet temporarily stored in the buffer.

\section{- $\quad$ Section \#2}

P-PEP 1 transmits data to P-PEP 2 via satellite. The flow controller of the satellite receiving the data measures the arrival time of the ACK message to be transmitted to the P-PEP 2. The ACK Controller then generates an ACK message, temporarily stores the packet in the buffer, and sends an ACK message to P-PEP 1. Then, it sends a packet to P-PEP 2. If the packet transmitted to the P-PEP 1 is a duplicate packet already stored in the buffer, the Flow Controller recognizes that the ACK message sent to the P-PEP 1 has not been normally transmitted and retransmits the ACK message in the buffer to the P-PEP 1 . If an ACK message is not received from the P-PEP 2 even after a predetermined time elapses, the packet corresponding to the P-PEP 2 is retransmitted. P-PEP 2, which receives data via satellite, converts the packet into a TCP structure through a converter. The ACK Controller generates an ACK message and transmits it to the satellite via the Flow Controller. The flow controller receiving the converted packet from the converter measures the arrival time of the ACK message for the packet, temporarily stores it in the data buffer, and transmits the packet to Host 2.

- Section \#3

Host 2, which has received the packet to P-PEP 2, transmits an ACK message. The P-PEP 2 Flow Controller retransmits the packet if an ACK message is not sent to Host 2 even after the expected ACK message arrival time measured in advance. Also, if the packet received from the satellite is a duplicate packet that is temporarily stored in the data buffer, it recognizes that there is a transmission failure between the satellite and the P-PEP 2 and retransmits the ACK for the packet stored in the buffer to the satellite.

\section{Conclusion}

In this paper, we propose a method to manage and manage each interval through ACK message when communicating through satellite. This makes it possible to transmit data more efficiently than the conventional method. However, in order to provide better quality service to users through satellite network, it is necessary to solve the problems such as path attenuation caused by long transmission and rain attenuation caused by weather deterioration such as rainy weather. 


\section{Acknowledgments}

This paper has been supported by the 2017 Hannam University Research Fund.

\section{References}

[1] J. Border, M. Kojo, J. Griner, G. Montenegro, Z. Shelby, "Performance Enhancing Proxies Intended to Mitigate Link-Related Degradations”, IETF RFC 3135, (2001) June.

[2] D. Lacamera, "PEPsal: A TCP Performance Enhancing Proxy for Satellite Links", Master in Free and Open Source Software Technologies, Almaweb Graduate School of Information Technology Management and Communication, (2005) May/June.

[3] K. Selén, "Evaluation of Performance Enhancing Proxies in a Global Network", Master's Thesis, Helsinki University of Technology, Faculty of Information and Natural Sciences, Department of Computer Science and Engineering, (2008) March 9.

[4] SatLabs, "INTEROPERABLE PEP (I-PEP), TRANSPORT EXTENSIONS AND SESSION FRAMEWORK FOR SATELLITE COMMUNICATIONS: AIR INTERFACE SPECIFICATION", http://satlabs.org/interoperability/interoperable-pep, (2005) October 27.

[5] B. I. Lee, Y. H. Won, H. Y. Song, H. K. Seo, "A Protocol Conversion Mechanism between Terrestrial and Satellite Communication Links", Korean Institute of Information Scientists and Engineers, vol. 31, no. 2, (2004). 
International Journal of $u-$ and $\mathrm{e}-$ Service, Science and Technology Vol.10, No.9 (2017) 